\title{
Spectroscopic distances of nearby ultracool dwarfs
}

\author{
N. Phan-Bao ${ }^{1}$ and M. S. Bessell ${ }^{2}$ \\ ${ }^{1}$ Institute of Astronomy and Astrophysics, Academia Sinica, PO Box 23-141, Taipei 106, Taiwan, R.O.C. \\ e-mail: pbngoc@asiaa.sinica.edu.tw \\ 2 Research School of Astronomy and Astrophysics, Australian National University, Cotter Rd, Weston, ACT 2611, Australia
}

Received 18 August 2005 / Accepted 29 September 2005

\section{ABSTRACT}

We present updated results of spectroscopic follow-up observations of a sample of $45 \mathrm{M}$ dwarf candidates identified by Phan-Bao et al. (2003, A\&A, 401, 959) based on the DENIS photometry and proper motion measurements. Forty one of these are nearby late-M dwarfs $(d<30 \mathrm{pc})$ with spectral types ranging from M5.0 to M8.5 computed from the spectral indices. One contaminant is probably an F-G main sequence star reddened by intervening dust and three stars that were not observed have previous classifications as $\mathrm{M}$ dwarfs in the literature. In this paper, we identify three M7.5, five M8.0, one M8.5 dwarf and confirm two new M8.0 dwarf members of the 25 pc volume.

Key words. stars: low-mass, brown dwarfs

\section{Introduction}

We present updated results of spectroscopic observations of nearby red dwarf candidates $(d<30 \mathrm{pc} ; 2 \leq I-J \leq 3)$ from Phan-Bao et al. (2001, 2003, hereafter Papers I and II respectively). These late-M dwarf candidates were either found in the DENIS survey (Epchtein et al. 1997) over 5700 square degrees, or cross-identified over a wider area between the DENIS database and the LHS or NLTT catalogs (Luyten 1979, 1980). All were further selected by the "Maximum Reduced Proper Motion" method (hereafter MRPM, see Paper II). The basic idea of this method is to use the reduced-proper-motion ( $\left.H=M+5 \log \left(V_{\mathrm{t}} / 4.74\right)\right)$ versus color diagram to distinguish between nearby ultra-cool dwarfs and more distant giants. This is done as follows: (1) calculate the maximum reduced-propermotion of a red giant at a given color - this corresponds to the largest possible tangential velocity, $V_{\mathrm{t}}$ of about $800 \mathrm{~km} \mathrm{~s}^{-1}$. (2) Any object with a reduced proper motion, calculated from the DENIS photometry and our proper motion measurements, larger than the maximum value of a red giant at the same color must be a dwarf.

That robust selection method retrieves solar neighbourhood late-M dwarfs down to very low proper motions (e.g., DENISP J1538317-103850, an M5.0 with $\mu=20$ mas/yr), well below the $0.18 \mathrm{arc}-\mathrm{sec} / \mathrm{yr}$ threshold of the NLTT catalog (Luyten 1980) and can therefore retrieve a good fraction of the estimated $26 \%$ nearby late-M dwarfs $(d<30 \mathrm{pc})$ missing from the NLTT catalog (Crifo et al. 2005).

The first spectroscopic observations of $32 \mathrm{M}$ dwarfs have been presented by Crifo et al. (2005) based on observations made at the ESO-1.52 m and NTT-3.6 m telescopes at La Silla,
Chile in 2002 and 2003. In this paper, we present spectroscopic observations of the remaining candidates made at the MSSSO-2.3 m telescope.

Section 2 describes our sample, spectroscopic observations and reductions. Section 3 presents the measurements of various spectroscopic indices, spectral type and distance estimates. Section 4 discusses the chromospheric activity in M dwarfs. We summarize our results in the final section.

\section{Data sample and spectroscopic observations}

\subsection{Data sample}

Papers I and II examined a total of 132 DENIS sources with $2 \leq I-J \leq 3$, and classified them into 80 probable dwarfs and 52 probable giants using the MRPM method. Crifo et al. (2005) observed 36 of the 80 dwarf candidates and confirmed 32 were $\mathrm{M}$ dwarfs and 4 were reddened F-K main sequence star contaminants. Phan-Bao et al. (2005) reported one object LP 714-37 as a binary system of very low mass stars. Our present sample includes the 43 remaining candidates plus two additional stars from the 52 probable giants list (Table 4, Paper II). These two candidates were fainter than the plate limit, so we could not measure their proper motion and they were therefore not ruled out by the MRPM. On the other hand, as they fell on the dwarf star sequence in the $(I-J, J-K)$ diagram (see Fig. 2, Paper I; Bessell \& Brett 1988) we added them to our spectroscopic sample list in order to confirm their nature. We finally had a total of 45 candidates. 


\subsection{Spectroscopic observations and reductions}

During our run, forty two of the stars were observed in July 2005 with the DBS spectrograph on the $2.3 \mathrm{~m}$ telescope at Siding Spring Observatory with the $158 \mathrm{~g} / \mathrm{mm}$ grating providing a wavelength coverage of $580-1030 \mathrm{~nm}$ at $0.5 \mathrm{~nm}$ resolution. Three remaining stars (LHS 2049, 5165 and LP 8591) were not been observed due to their having too large an airmass, however, these stars have previous classifications as M dwarfs in the literature.

The data were reduced using FIGARO. Smooth spectrum stars were observed at a range of airmass to remove the telluric lines as described in Bessell (1999) and the spectrophotometric standard EG131 (loc cit) was used to put the data on a relative absolute flux scale. A NeAr arc was used for wavelength calibration.

All spectra were normalized over the $754-758 \mathrm{~nm}$ interval that is the denominator of the PC3 index and a region with a good flat pseudo-continuum.

At the resolution of these spectra, the presence of the NaI and $\mathrm{KI}$ doublets, the presence of $\mathrm{FeH}$ and the appearance of strong $\mathrm{CaH}$ cutting into the continuum shortward of $700 \mathrm{~nm}$ and the absence of the CaII triplet immediately distinguish M dwarfs from M giants (see Bessell 1991).

One of the 42 observed targets, LP 862-111 (or J1641-2359), has a much earlier spectrum indicating that it has been reddened by intervening dust as discussed in Crifo et al. (2005). Blue spectra would be needed for precise classification at earlier spectral types, but from the strength of the Paschen lines the star is probably an F-G dwarf (Fig. 5).

Figure 1 shows the 41 spectra of nearby late-M dwarfs and the VB 10 (M8.0) standard obtained at the MSSSO-2.3 m telescope.

\section{Spectroscopic indices, spectral type classifications and distances}

In the past decade, many important spectral indices have been defined for M and L dwarfs (e.g., Reid et al. 1995; Martín et al. 1999; Kirkpatrick et al. 1999; Lépine et al. 2003a). These indices allow us to quantify spectral types (e.g., PC, TiO, and VO) and metallicity classes (e.g., $\mathrm{CaH}$ ) of red dwarfs.

For the work here, we have used some available indices to estimate spectral types and distinguish between $\mathrm{M}$ dwarfs and (e)subdwarfs in our sample. Table 1 lists our spectral indices measurement. For classifying spectral types of $\mathrm{M}$ dwarfs, we use the PC3 index defined by Martín et al. (1999), TiO5 in Reid et al. (1995) and VOa in Kirkpatrick et al. (1999). The final spectral type is an average value of three spectral types computed from these three indices, except some cases as discussed below.

Figure 2 shows the comparison between the $\mathrm{CaH}$ and $\mathrm{TiO} 5$ indices measured from our observations of DENIS nearby candidates and also plots dwarfs and metal-poor dwarfs from Gizis (1997). Two stars: J0312+0021 and J1512-1032, are very faint with low signal-to-noise spectra and could be mild subdwarfs. However, the combination of two diagrams: TiO5 vs. $\mathrm{CaH} 2$ and $\mathrm{TiO} 5$ vs. $(\mathrm{CaH} 1+\mathrm{CaH} 2)$, does not support $\mathrm{J} 0312+0021$ being a subdwarf, and comparison of the spectra of J1512-1032, the standard VB 10 and an M8.0 subdwarf template (LSR 1425+7102) from Sébastien Lépine clearly shows that J1512-1032 is a late-M dwarf rather than a subdwarf (Fig. 3).

In fact, comparison of all our spectra with M subdwarf references (e.g., Bessell 1982; Gizis 1997) does not reveal any subdwarfs in our sample. This is not unexpected, as extreme subdwarfs are intrinsically very faint and rare and our volume size is limited and too small to have much probability of containing extreme halo members.

Some extreme $\mathrm{M}$ subdwarf spectra are given in Bessell (1982), amongst which are the faint pair LHS $2099(R \sim 16)$ and LHS $2100(R \sim 19)$. The spectrum of LHS 2099 is identical to that of LHS $541(R \sim 15)$, the M subdwarf companion of the well known $[\mathrm{Fe} / \mathrm{H}]=-1.7$ subdwarf HD $219617(R \sim 8)$. LHS 2100 is the best example of a very cool $[\mathrm{Fe} / \mathrm{H}]=-1.7$ M subdwarf.

Table 2 lists our spectral type estimates by using the formulae given in Martín et al. (1999) for the PC3 index and Cruz \& Reid (2002) for the TiO5 and VOa indices. To avoid saturation in TiO5 around about spectral type M7, we firstly computed spectral types using the PC3 index. We then used Cruz \& Reid's formula: $S_{\mathrm{p}}=-10.775(\mathrm{TiO} 5)+8.200$ for spectral types later than M7 and $S_{\mathrm{p}}=5.673(\mathrm{TiO} 5)+6.221$ for earlier ones. We adopted the mean spectral types computed from these three indices with an uncertainty of \pm 0.5 subclass. One should note for J1136-0755 that our spectral type estimate from TiO5 is M2.7, however comparison of its spectrum with early-M dwarf templates in Bessell (1991) indicates clearly that this is a mid-M dwarf rather than an early$\mathrm{M}$ dwarf. This is consistent with spectral types estimated from PC3 and VOa and we finally adopted a spectral type of M6.0 for J1136-0755 (or LP 673-63). In this paper, we found three M7.5 dwarfs: J0041-5621, J0145-3729, J1357-1438; five M8.0: J0120-0741, J0517-3349, J1021-2044, J1141-2232, J1512-1032; one M8.5: J2353-0833, the latest in our sample is at $19 \mathrm{pc}$. All of them have proper motions measured in Paper II, except J1021-2044 and J1512-1032 that were below the plate limit plate and are located in the $25 \mathrm{pc}$ volume.

To estimate the distances, we used the magnitude versus PC3 index relations given in Crifo et al. (2005) with a $\sim 12 \%$ error in the distance to single stars. Table 2 lists the absolute magnitudes in the three DENIS bands computed from the PC3 index, as well as the estimated spectrophotometric distances for each of those bands and their average. The values for the three colours $I, J, K$ are very similar, indicating correlated uncertainties for the three estimators. For unresolved binaries in our sample, their distances are underestimated by up to $\sqrt{2}$. We also list in the table our distances estimated from the $I-J$ color to I-band absolute magnitude relation given in Paper II. The distances computed from 4 estimators are reasonably well matched. In this table, there are two new M8.0 members of the 25 pc volume: J1021-2044 and J1512-1032. 
N. Phan-Bao and M. S. Bessell: Nearby ultracool dwarfs

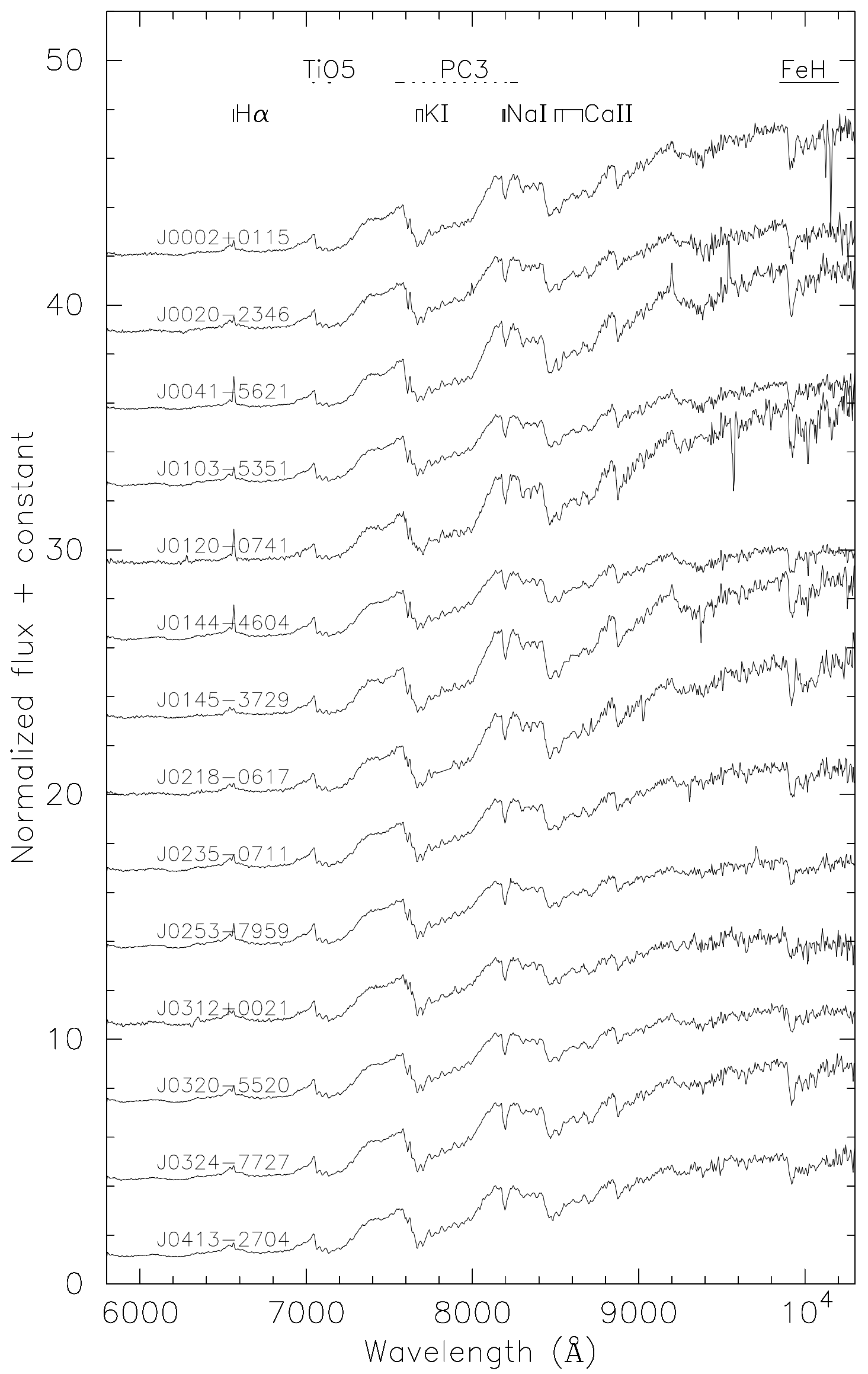

Fig. 1. Spectra of the 41 late-M dwarfs and VB 10. The positions of the $\mathrm{H}_{\alpha}, \mathrm{NaI}, \mathrm{KI}$ and CaII lines are indicated, as well as the spectral intervals used to compute the TiO5, and PC3 indices. The FeH absorption features from 985 to $1020 \mathrm{~nm}$ are also indicated. 


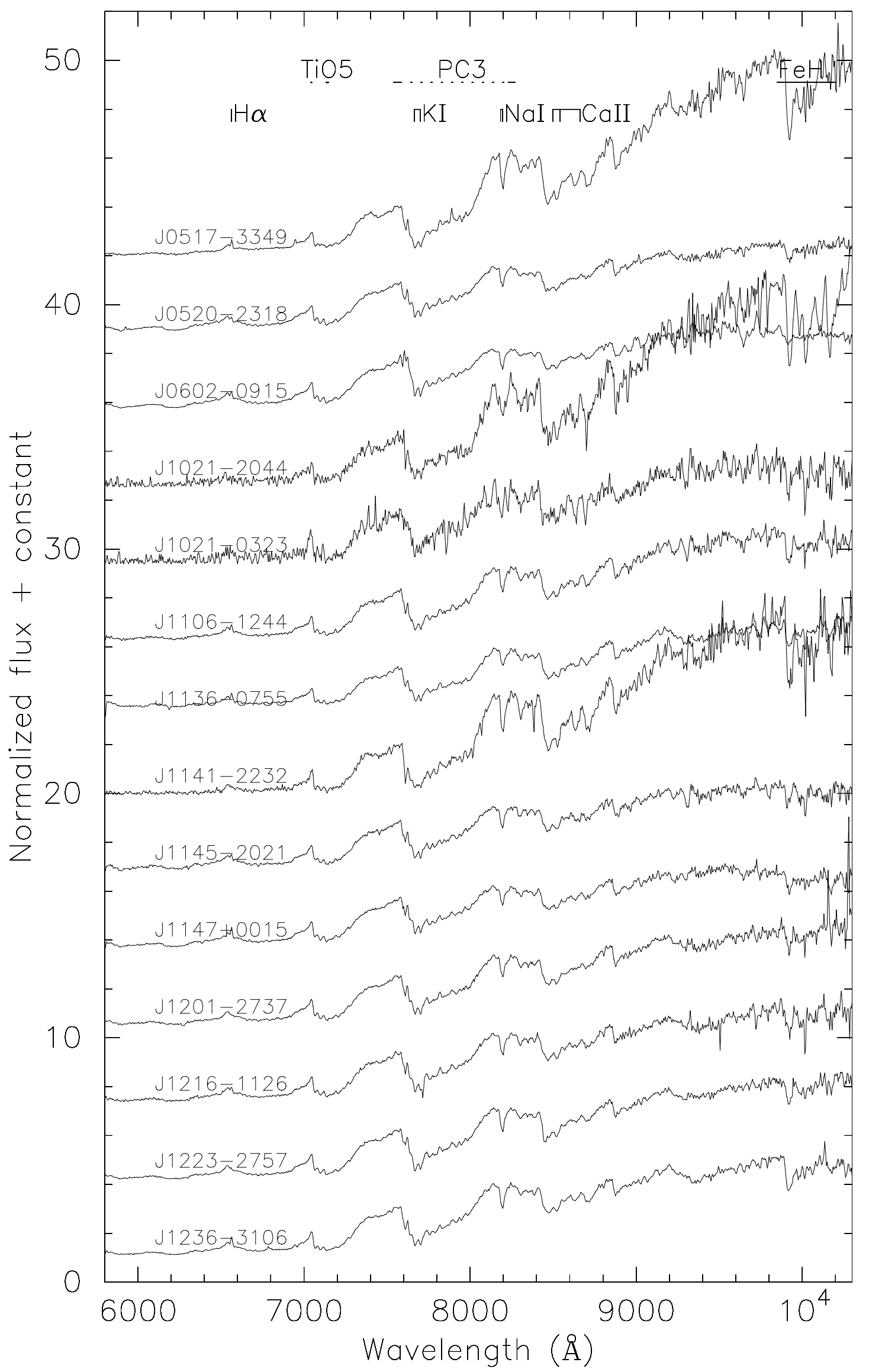

Fig. 1. continued. 
N. Phan-Bao and M. S. Bessell: Nearby ultracool dwarfs

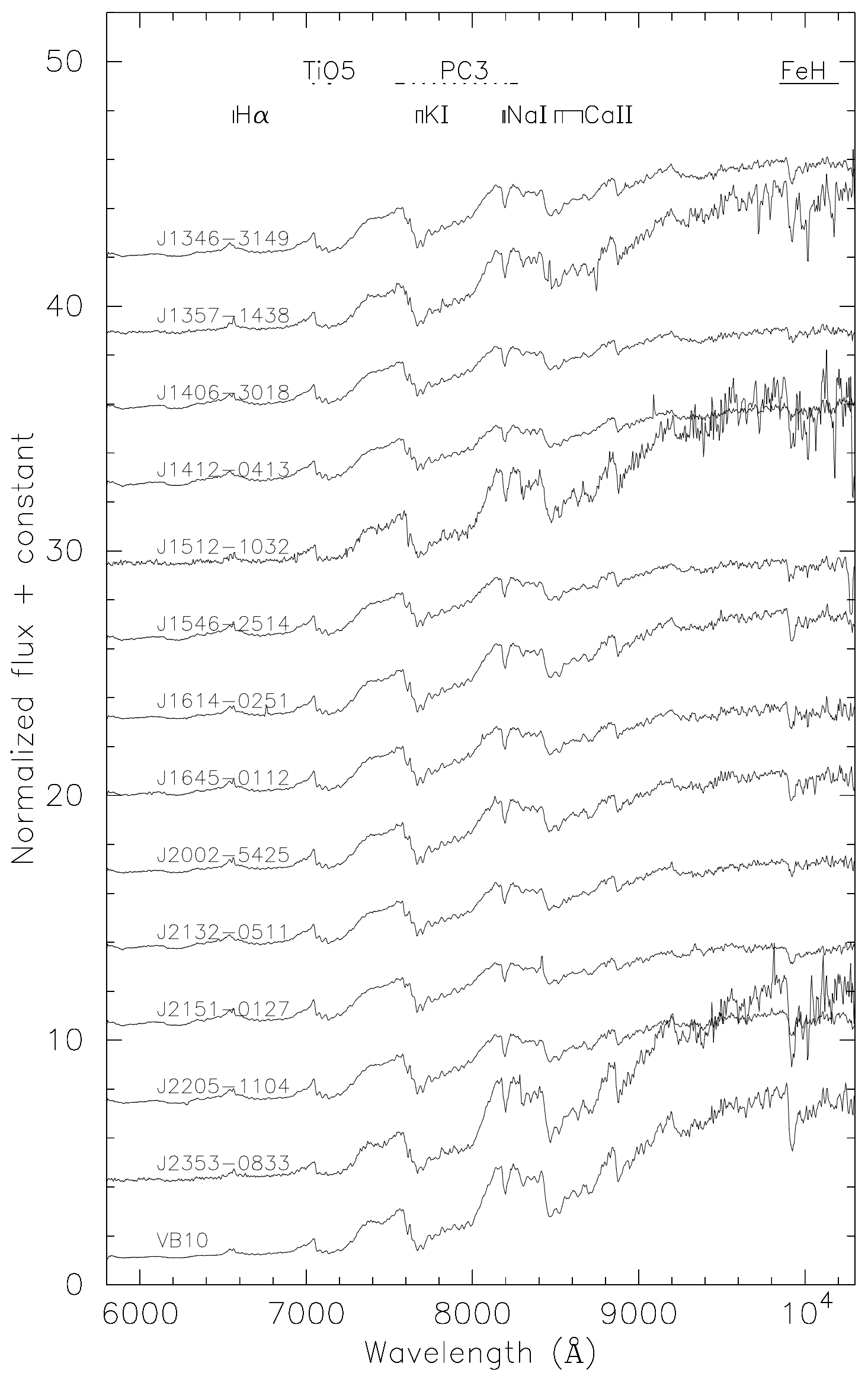

Fig. 1. continued. 
Table 1. Spectral indices for the 41 nearby red dwarfs and the VB 10 standard observed at MSSSO-2.3 m.

\begin{tabular}{|c|c|c|c|c|c|c|c|c|c|c|c|c|}
\hline Stars & $\begin{array}{l}\text { NLTT/LHS } \\
\text { name } \\
\text { (2) }\end{array}$ & $\mathrm{H}_{\alpha}$ & PC3 & $\mathrm{CrH} 1$ & $\mathrm{CrH} 2$ & $\mathrm{FeH} 1$ & $\mathrm{FeH} 2$ & $\mathrm{CaH} 1$ & $\mathrm{CaH} 2$ & $\mathrm{CaH} 3$ & $\mathrm{TiO} 5$ & VOa \\
\hline J0002061+011536 & LP 584-4 & 1.35 & 1.60 & 0.97 & 1.15 & 0.95 & 1.32 & 0.842 & 0.276 & 0.608 & 0.265 & 2.20 \\
\hline J0020231-234605 & LP 825-35 & 1.26 & 1.51 & 0.98 & 1.12 & 0.96 & 1.29 & 0.933 & 0.283 & 0.615 & 0.297 & 2.12 \\
\hline J0041353-562112 & $\ldots$ & 2.83 & 1.69 & 0.95 & 1.13 & 0.89 & 1.31 & 0.910 & 0.269 & 0.613 & 0.269 & 2.27 \\
\hline J0103119-535143 & $\ldots$ & 1.49 & 1.44 & 0.97 & 1.07 & 0.94 & 1.21 & 0.882 & 0.300 & 0.620 & 0.297 & 2.12 \\
\hline J0120491-074103 & $\ldots$ & 2.70 & 1.80 & 0.99 & 1.14 & 0.94 & 1.22 & 1.134 & 0.289 & 0.583 & 0.307 & 2.31 \\
\hline J0144318-460432 & $\ldots$ & 2.24 & 1.38 & 0.94 & 1.09 & 0.91 & 1.25 & 0.832 & 0.281 & 0.584 & 0.265 & 2.08 \\
\hline J0145434-372959 & & 1.03 & 1.75 & 0.94 & 1.14 & 0.88 & 1.22 & 0.801 & 0.254 & 0.540 & 0.250 & 2.23 \\
\hline J0218579-061749 & LP 649-93 & 1.23 & 1.64 & 0.98 & 1.16 & 0.91 & 1.22 & 0.798 & 0.261 & 0.612 & 0.235 & 2.22 \\
\hline J0235495-071121 & $\ldots$ & 1.25 & 1.47 & 0.99 & 1.15 & 0.96 & 1.24 & 0.840 & 0.295 & 0.584 & 0.287 & 2.11 \\
\hline J0253444-795913 & $\ldots$ & 1.60 & 1.38 & 0.97 & 1.12 & 0.98 & 1.23 & 0.795 & 0.262 & 0.566 & 0.270 & 2.05 \\
\hline J0312251+002158 & $\ldots$ & 1.11 & 1.37 & 0.99 & 1.13 & 1.00 & 1.25 & 0.643 & 0.300 & 0.582 & 0.295 & 2.10 \\
\hline J0320588-552015 & $\ldots$ & 1.27 & 1.42 & 0.97 & 1.08 & 0.96 & 1.19 & 0.848 & 0.303 & 0.610 & 0.293 & 2.09 \\
\hline J0324268-772705 & & 1.16 & 1.56 & 0.98 & 1.18 & 1.00 & 1.32 & 0.821 & 0.268 & 0.540 & 0.259 & 2.08 \\
\hline J0413398-270428 & LP 890-2 & 1.23 & 1.43 & 0.96 & 1.15 & 0.96 & 1.20 & 0.862 & 0.279 & 0.590 & 0.252 & 2.11 \\
\hline J0517377-334903 & $\ldots$ & 1.34 & 2.08 & 1.03 & 1.26 & 1.04 & 1.41 & 0.913 & 0.350 & 0.605 & 0.394 & 2.22 \\
\hline J0520293-231848 & LP 836-41 & 1.02 & 1.31 & 0.95 & 1.06 & 0.92 & 1.15 & 0.875 & 0.348 & 0.687 & 0.317 & 2.06 \\
\hline J0602542-091503 & LHS 1810 & 1.18 & 1.20 & 0.97 & 1.10 & 0.97 & 1.16 & 0.859 & 0.273 & 0.604 & 0.278 & 2.07 \\
\hline J1021323-204407 & $\ldots$ & 0.67: & 2.06 & 1.10 & 1.22 & 1.09 & 1.32 & 1.612: & 0.210: & 0.528 & $0.264:$ & 2.23 \\
\hline J1021513-032309 & LP 610-5 & 0.86 : & 1.42 & 1.08 & 1.11 & 1.03 & 1.25 & 0.421: & 0.212 & 0.435 & 0.081: & 1.96: \\
\hline J1106569-124402 & LP 731-47 & 1.25 & 1.45 & 0.98 & 1.14 & 0.97 & 1.24 & 0.884 & 0.265 & 0.565 & 0.258 & 2.15 \\
\hline J1136409-075511 & LP 673-63 & 1.17 & 1.37 & 0.96 & 1.07 & 0.91 & 1.18 & 0.923 & 0.541 & 0.724 & 0.507 & 2.12 \\
\hline J1141440-223215 & $\ldots$ & 0.90 & 2.01 & 1.00 & 1.26 & 1.03 & 1.42 & $0.678:$ & 0.245 & 0.467 & $0.188:$ & 2.20 \\
\hline J1145354-202105 & LP 793-34 & 1.10 & 1.30 & 0.99 & 1.16 & 0.99 & 1.17 & 0.766 & 0.310 & 0.601 & 0.314 & 2.09 \\
\hline $\mathrm{J} 1147421+001506$ & LP 613-50 & 1.32 & 1.22 & 0.96 & 1.11 & 0.94 & 1.20 & 0.772 & 0.313 & 0.621 & 0.295 & 2.06 \\
\hline J1201421-273746 & LP 908-5 & 0.86 & 1.39 & 0.92 & 1.10 & 0.89 & 1.16 & 0.892 & 0.272 & 0.588 & 0.257 & 2.10 \\
\hline J1216101-112609 & LP 734-87 & 1.08 & 1.37 & 0.99 & 1.18 & 0.98 & 1.27 & 0.881 & 0.281 & 0.570 & 0.275 & 2.10 \\
\hline $\mathrm{J} 1223562-275746$ & LHS 325a & 0.91 & 1.42 & 0.95 & 1.10 & 0.94 & 1.18 & 0.869 & 0.254 & 0.582 & 0.233 & 2.12 \\
\hline J1236153-310646 & LP 909-55 & 1.36 & 1.44 & 0.99 & 1.19 & 1.01 & 1.36 & 0.790 & 0.279 & 0.498 & 0.271 & 2.08 \\
\hline J1346460-314925 & LP 911-56 & 0.92 & 1.44 & 0.95 & 1.08 & 0.93 & 1.19 & 0.873 & 0.267 & 0.594 & 0.242 & 2.12 \\
\hline J1357149-143852 & $\ldots$ & 1.52 & 1.72 & 1.01 & 1.30 & 1.01 & 1.35 & 0.959 & 0.300 & 0.615 & 0.316 & 2.20 \\
\hline J1406493-301828 & LHS 2859 & 1.17 & 1.29 & 0.96 & 1.04 & 0.92 & 1.12 & 0.862 & 0.348 & 0.662 & 0.322 & 2.05 \\
\hline J1412069-041348 & LP 679-32 & 0.91 & 1.26 & 0.94 & 1.05 & 0.89 & 1.09 & 0.907 & 0.373 & 0.711 & 0.345 & 2.07 \\
\hline J1512333-103241 & $\ldots$ & 1.06: & 1.94 & 0.99 & 1.24 & 1.00 & 1.35 & $0.824:$ & 0.246 & 0.650 & 0.340 & 2.29 \\
\hline J1546115-251405 & LP 860-30 & 1.25 & 1.30 & 0.96 & 1.10 & 0.94 & 1.20 & 0.836 & 0.341 & 0.651 & 0.304 & 2.04 \\
\hline $\mathrm{J} 1614252-025100$ & LP $624-54$ & 1.12 & 1.52 & 0.95 & 1.12 & 0.95 & 1.24 & 0.843 & 0.270 & 0.583 & 0.249 & 2.12 \\
\hline J1645282-011228 & LP 626-2 & 1.22 & 1.39 & 0.97 & 1.09 & 0.96 & 1.20 & 0.852 & 0.296 & 0.577 & 0.271 & 2.06 \\
\hline J2002134-542555 & $\ldots$ & 1.25 & 1.48 & 0.96 & 1.13 & 0.98 & 1.27 & 0.783 & 0.257 & 0.562 & 0.250 & 2.10 \\
\hline $\mathrm{J} 2132297-051158$ & LP 698-2 & 0.89 & 1.34 & 0.93 & 1.04 & 0.89 & 1.10 & 0.865 & 0.340 & 0.694 & 0.294 & 2.08 \\
\hline $\mathrm{J} 2151270-012713$ & LP 638-50 & 1.20 & 1.29 & 0.97 & 1.10 & 0.98 & 1.18 & 0.796 & 0.308 & 0.599 & 0.304 & 2.05 \\
\hline J2205357-110428 & LP 759-25 & 1.10 & 1.41 & 0.97 & 1.12 & 0.98 & 1.23 & 0.819 & 0.276 & 0.562 & 0.280 & 2.08 \\
\hline J2353594-083331 & $\ldots$ & 1.33 & 2.07 & 0.99 & 1.27 & 0.96 & 1.45 & 0.876 & 0.348 & 0.679 & 0.356 & 2.29 \\
\hline VB10 & LHS 474 & 1.09 & 1.87 & 0.96 & 1.14 & 0.92 & 1.32 & 0.939 & 0.318 & 0.633 & 0.307 & 2.32 \\
\hline
\end{tabular}

Columns 1 and 2: full DENIS name with the DENIS-P prefix and NLTT/LHS name.

Columns 3-13: Spectroscopic indices. $\mathrm{H}_{\alpha}$ defined in Reid et al. (1995); PC3, CrH1, CrH2, FeH1, FeH2 in Martín et al. (1999); CaH1, CaH2, CaH3, TiO5 in Reid et al. (1995); VOa in Kirkpatrick et al. (1999). A colon (“:”) indicates unreliable values due to low signal-to-noise spectra.

\section{Discussion}

Seventy eight percent of the stars in our sample exhibited $\mathrm{H}_{\alpha}$ emission. We directly measured $\mathrm{H}_{\alpha}$ equivalent widths using the IRAF task SPLOT. Table 2 (Col. 15) lists our measurements for 41 stars. We also measured an upper limit for the remaining stars which have a weak $\mathrm{H}_{\alpha}$ emission or low signal-to-noise spectra. Note that there are two late-M dwarfs exhibiting very strong $\mathrm{H}_{\alpha}$ emission: J0041-5621 (M7.5, EW = 37.1) and J0120-0741 (M8.0, EW = 51.3).

It is interesting to note that a flaring state was seen in the spectrum of LP 890-2 (or J0413-2704, M6.0) obtained by Cruz \& Reid (2002) in comparison with ours. This M6.0 dwarf showed strong $\mathrm{H}_{\alpha}$ emission $(E W=16.0 \AA)$ in Cruz and Reid's observation (their Fig. 7) but is much weaker in ours $(E W=5.0 \AA)$. Figure 4 plots $\mathrm{H}_{\alpha}$ equivalent widths versus 

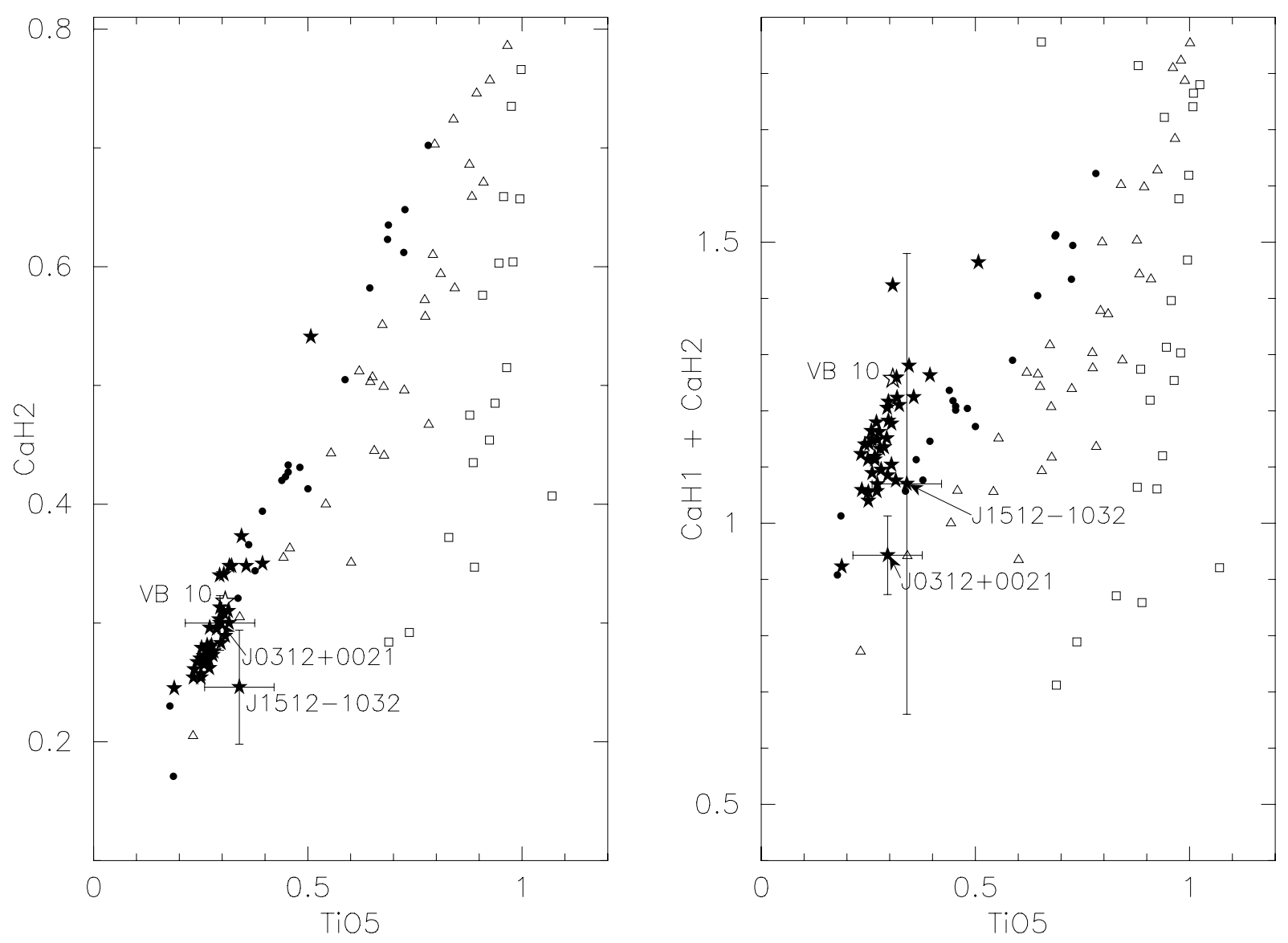

Fig. 2. $\mathrm{CaH}$ vs. TiO5 diagrams. The filled stars represent the measurements of this paper. Solid circles are M dwarfs, open triangles are sdM subdwarfs and open squares are esdM subdwarfs from Gizis (1997). The empty star is VB 10. Two possible M subdwarfs are indicated: DENIS-P J0312+0021 and J1512-1032. In the right panel DENIS-P J0312+0021 appears a probable M subdwarf due to large error bars, however the $\mathrm{CaH} 1$ vs. TiO5 diagram indicates that DENIS-P J0312+0021 is well located on the dwarf sequence and it is ruled out as metal poor dwarf. Comparison of spectra between DENIS-P J1512-1032 and subdwarf templates also confirms it to be a late-M dwarf, see Fig. 3. A few stars with unreliable spectral indices are not plotted (Table 1), see discussion in Sect. 3.

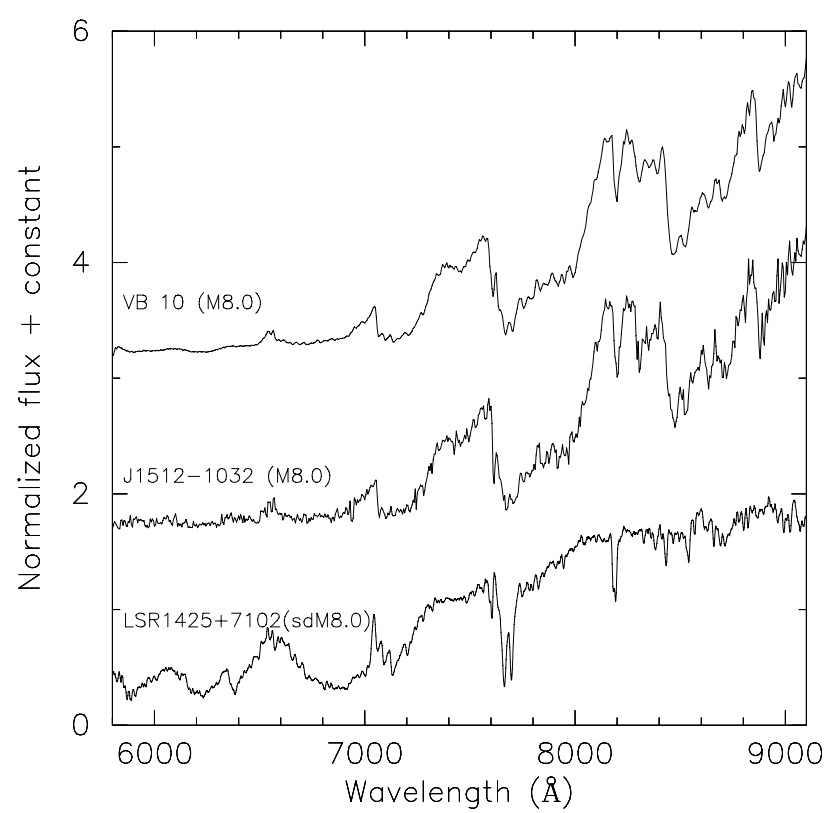

Fig. 3. Comparison between spectra of DENIS-P J 1512-1032 (M8.0) and an M8.0 dwarf (VB 10, top) and an M8.0 subdwarf template (LSR 1425+7102, Lépine et al. 2003b).

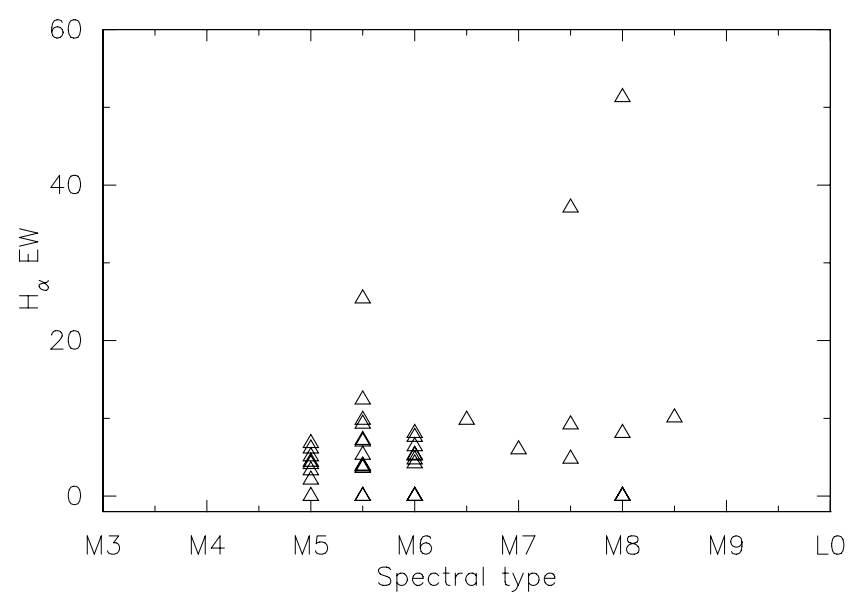

Fig. 4. $\mathrm{H}_{\alpha}$ equivalent width vs. spectral type diagram for all stars with spectral types computed in this paper.

spectral types. One M8.0 dwarf (J1141-2232) has no emission or too weak $\mathrm{H}_{\alpha}$ emission, we set an upper limit of $0.8 \AA$ for this late-M star.

We note that one of our targets DENIS-P J1614-2359 (LP 826-111) is a reddened F-G main sequence star. Figure 5 
Table 2. Estimated absolute magnitude, spectrophotometric distance and spectral type for the 41 nearby M dwarfs and VB 10 (M8.0).

\begin{tabular}{|c|c|c|c|c|c|c|c|c|c|c|c|c|c|c|}
\hline Stars & $\begin{array}{l}R-I \\
(2)\end{array}$ & $M_{I}$ & $M_{J}$ & $M_{K}$ & $\begin{array}{l}d_{I} \\
(6) \\
\end{array}$ & $d_{J}$ & $d_{K}$ & $\begin{array}{l}d_{\mathrm{sp}} \\
(9) \\
\end{array}$ & $\begin{array}{l}d \\
(\mathrm{IJ}) \\
(10)\end{array}$ & $\begin{array}{l}\text { Sp. T } \\
\text { VOa } \\
(11)\end{array}$ & $\begin{array}{l}\text { Sp. T } \\
\text { TiO5 } \\
(12)\end{array}$ & $\begin{array}{l}\text { Sp. T } \\
\text { PC3 } \\
(13)\end{array}$ & $\begin{array}{l}\text { Sp. T } \\
\text { adopted } \\
(14)\end{array}$ & $\begin{array}{l}E W \\
\mathrm{H}_{\alpha}(\AA) \\
(15)\end{array}$ \\
\hline $\mathrm{J} 0002+0115$ & 2.55 & 13.13 & 10.62 & 9.65 & 21.6 & 21.4 & 20.0 & 21.0 & 20.4 & M6.9 & M5.3 & M6.9 & M6.5 & 9.8 \\
\hline J0020-2346 & 2.48 & 12.77 & 10.43 & 9.51 & 23.7 & 23.3 & 22.6 & 23.2 & 21.4 & M6.0 & M5.0 & M6.4 & M6.0 & 8.1 \\
\hline J0041-5621 & 2.60 & 13.46 & 10.79 & 9.78 & 17.6 & 16.9 & 16.5 & 17.0 & 16.5 & M7.6 & M7.7 & M7.3 & M7.5 & 37.1 \\
\hline J0103-5351 & 2.41 & 12.49 & 10.26 & 9.38 & 25.8 & 25.2 & 21.9 & 24.3 & 23.1 & M6.0 & M5.0 & M6.0 & M5.5 & 9.8 \\
\hline J0120-0741 & 2.57 & 13.84 & 11.00 & 9.93 & 23.7 & 24.5 & 23.1 & 23.8 & 26.0 & M8.0 & M8.0 & M7.8 & M8.0 & 51.3 \\
\hline J0144-4604 & 2.38 & 12.23 & 10.11 & 9.25 & 23.7 & 22.9 & 23.4 & 23.3 & 20.8 & M5.6 & M5.3 & M5.6 & M5.5 & 25.4 \\
\hline J0145-3729 & 2.62 & 13.67 & 10.91 & 9.86 & 18.9 & 20.7 & 21.7 & 20.5 & 22.3 & M7.2 & M7.6 & M7.6 & M7.5 & 4.8 \\
\hline $\mathrm{J} 0218-0617^{c}$ & 2.57 & 13.27 & 10.70 & 9.71 & 28.6 & 27.7 & 24.9 & 27.1 & 26.4 & M7.1 & M7.6 & M7.1 & M7.0 & 6.0 \\
\hline J0235-0711 & 2.41 & 12.61 & 10.34 & 9.43 & 26.3 & 25.8 & 24.5 & 25.5 & 23.6 & M5.9 & M5.1 & M6.2 & M5.5 & 7.2 \\
\hline J0253-7959 & 2.36 & 12.23 & 10.11 & 9.25 & 17.7 & 17.4 & 16.5 & 17.2 & 16.4 & M5.3 & M5.3 & M5.6 & M5.5 & 12.4 \\
\hline J0312+0021 & 2.34 & 12.18 & 10.08 & 9.23 & 26.5 & 25.6 & 24.8 & 25.7 & 23.4 & M5.8 & M5.0 & M5.6 & M5.5 & 3.9 \\
\hline J0320-5520 & 2.38 & 12.40 & 10.21 & 9.34 & 24.0 & 23.5 & 21.9 & 23.1 & 21.7 & M5.7 & M5.0 & M5.9 & M5.5 & 7.0 \\
\hline J0324-7727 & 2.49 & 12.97 & 10.54 & 9.59 & 19.0 & 20.6 & 19.7 & 19.7 & 21.5 & M5.6 & M5.4 & M6.7 & M6.0 & 4.7 \\
\hline $\mathrm{J} 0413-2704^{d}$ & 2.42 & 12.44 & 10.24 & 9.36 & 25.2 & 24.6 & 23.4 & 24.4 & 22.4 & M5.9 & M5.5 & M5.9 & M6.0 & 5.2 \\
\hline J0517-3349 & 2.62 & 14.70 & 11.52 & 10.34 & 11.1 & 12.7 & 12.6 & 12.1 & 16.4 & M7.1 & M8.5 & M8.9 & M8.0 & 8.1 \\
\hline J0520-2318 & 2.26 & 11.91 & 9.92 & 9.08 & 26.4 & 23.3 & 20.4 & 23.4 & 18.2 & M5.4 & M4.8 & M5.2 & M5.0 & 2.1 \\
\hline $\mathrm{J} 0602-0915^{c}$ & 2.26 & 11.39 & 9.57 & 8.76 & 20.8 & 18.1 & 17.8 & 18.9 & 13.7 & M5.5 & M5.2 & M4.5 & M5.0 & 4.4 \\
\hline J1021-2044 & 2.60 & 14.64 & 11.48 & 10.31 & 19.5 & 21.2 & 21.8 & 20.8 & 25.6 & M7.2 & M7.7: & M8.9 & M8.0 & $<2.9$ \\
\hline $\mathrm{J} 1021-0323^{d}$ & 2.39 & 12.40 & 10.21 & 9.34 & 26.9 & 25.4 & 25.4 & 25.9 & 22.2 & M4.3: & M7.3: & M5.9 & M6.0 & $<3.0$ \\
\hline J1106-1244 & 2.46 & 12.53 & 10.29 & 9.40 & 21.4 & 19.8 & 20.5 & 20.5 & 16.9 & M6.3 & M5.4 & M6.0 & M6.0 & 6.4 \\
\hline J1136-0755 & 1.99 & 12.18 & 10.08 & 9.23 & 26.6 & 25.4 & 24.9 & 25.7 & 22.6 & M6.0 & M2.7: & M5.6 & M6.0 & 5.3 \\
\hline J1141-2232 & 2.69 & 14.50 & 11.38 & 10.22 & 15.3 & 18.3 & 17.9 & 17.2 & 23.6 & M6.9 & M7.3: & M8.7 & M8.0 & $<0.8$ \\
\hline J1145-2021 & 2.29 & 11.87 & 9.89 & 9.06 & 24.8 & 22.9 & 22.4 & 23.4 & 19.5 & M5.7 & M4.8 & M5.1 & M5.0 & 3.3 \\
\hline $\mathrm{J} 1147+0015$ & 2.26 & 11.48 & 9.64 & 8.83 & 21.9 & 19.9 & 18.6 & 20.1 & 16.5 & M5.4 & M5.0 & M4.6 & M5.0 & 6.8 \\
\hline $\mathrm{J} 1201-2737^{c}$ & 2.41 & 12.27 & 10.14 & 9.27 & 25.5 & 24.6 & 24.1 & 24.7 & 22.2 & M5.8 & M5.4 & M5.7 & M5.5 & $<0.6$ \\
\hline J1216-1126 & 2.40 & 12.18 & 10.08 & 9.23 & 32.2 & 29.3 & 26.2 & 29.2 & 24.3 & M5.8 & M5.2 & M5.6 & M5.5 & 3.8 \\
\hline J1223-2757 & 2.46 & 12.40 & 10.21 & 9.34 & 22.8 & 21.6 & 21.6 & 22.0 & 19.0 & M6.0 & M5.7 & M5.9 & M6.0 & $<0.9$ \\
\hline J1236-3106 & 2.42 & 12.49 & 10.26 & 9.38 & 19.8 & 19.7 & 18.6 & 19.4 & 18.6 & M5.6 & M5.3 & M6.0 & M5.5 & 9.3 \\
\hline J1346-3149 & 2.45 & 12.49 & 10.26 & 9.38 & 14.4 & 14.2 & 13.1 & 13.9 & 13.3 & M6.0 & M5.6 & M6.0 & M6.0 & $<0.5$ \\
\hline J1357-1438 & 2.55 & 13.56 & 10.85 & 9.82 & 25.0 & 25.2 & 23.9 & 24.7 & 25.6 & M6.9 & M8.0 & M7.5 & M7.5 & 9.2 \\
\hline $\mathrm{J} 1406-3018^{c}$ & 2.25 & 11.82 & 9.86 & 9.03 & 20.9 & 19.3 & 17.8 & 19.3 & 16.5 & M5.3 & M4.7 & M5.1 & M5.0 & 4.1 \\
\hline $\mathrm{J} 1412-0413^{c}$ & 2.20 & 11.68 & 9.77 & 8.95 & 24.9 & 23.5 & 21.8 & 23.4 & 21.2 & M5.5 & M4.5 & M4.9 & M5.0 & $<0.6$ \\
\hline J1512-1032 & 2.63 & 14.29 & 11.25 & 10.12 & 22.0 & 23.4 & 22.9 & 22.8 & 26.6 & M7.8 & M8.1 & M8.4 & M8.0 & $<5.0$ \\
\hline J1546-2514 & 2.25 & 11.87 & 9.89 & 9.06 & 27.8 & 26.5 & 25.8 & 26.7 & 23.8 & M5.2 & M4.9 & M5.1 & M5.0 & 6.1 \\
\hline J1614-0251 & 2.50 & 12.81 & 10.45 & 9.52 & 14.6 & 15.1 & 14.3 & 14.6 & 14.8 & M6.0 & M5.5 & M6.4 & M6.0 & 4.2 \\
\hline J1645-0112 & 2.38 & 12.27 & 10.14 & 9.27 & 25.2 & 25.1 & 24.3 & 24.9 & 24.2 & M5.4 & M5.3 & M5.7 & M5.5 & 5.3 \\
\hline J2002-5425 & 2.46 & 12.65 & 10.36 & 9.45 & 17.7 & 18.5 & 16.3 & 17.5 & 18.6 & M5.8 & M5.5 & M6.2 & M6.0 & 7.6 \\
\hline $\mathrm{J} 2132-0511^{c}$ & 2.29 & 12.05 & 10.00 & 9.16 & 19.7 & 19.0 & 16.7 & 18.5 & 17.5 & M5.6 & M5.0 & M5.4 & M5.5 & $<1.1$ \\
\hline $\mathrm{J} 2151-0127^{c}$ & 2.26 & 11.82 & 9.86 & 9.03 & 19.0 & 18.5 & 18.6 & 18.7 & 17.8 & M5.3 & M4.9 & M5.1 & M5.0 & 5.1 \\
\hline $\mathrm{J} 2205-1104^{c}$ & 2.38 & 12.36 & 10.19 & 9.32 & 18.3 & 18.6 & 17.7 & 18.2 & 18.5 & M5.6 & M5.2 & M5.8 & M5.5 & 3.6 \\
\hline J2353-0833 & 2.64 & 14.67 & 11.50 & 10.32 & 17.8 & 19.6 & 20.0 & 19.1 & 24.0 & M7.8 & M8.2 & M8.9 & M8.5 & 10.1 \\
\hline VB 10 & 2.62 & $14.07^{b}$ & $11.12^{b}$ & $10.02^{b}$ & 5.7 & 5.8 & 5.7 & $5.7^{a}$ & 6.3 & M8.1 & M8.0 & M8.1 & M8.0 & 3.8 \\
\hline
\end{tabular}

${ }^{a} d_{\pi}=5.87 \mathrm{pc}$, derived from $\pi=170.3$ mas, a companion of HIP $94761 ;{ }^{b}$ optical and infrared photometry from Bessell (1991); ${ }^{c}$ also listed by Reid et al. (2003); ${ }^{d}$ see Cruz et al. (2003).

Column 1: abbreviated DENIS name; Col. 2: $R-I$ color computed from spectra; Cols. 3-5: absolute magnitudes for the $I, J, K$ bands based on the PC3-absolute magnitudes relation; Cols. 6-8: distance (pc) estimated from the DENIS photometry and the $M_{I}, M_{J}, M_{K}$ derived from the PC3 index; Cols. 9 and 10: adopted distance, and distance previously derived in Paper II from the $I-J$ colour; Cols. 11-14: spectral types derived from the VOa, TiO5, and PC3 index using the formula given in Cruz \& Reid (2002) and Martín et al. (1999), and the adopted spectral rounded to the nearest half subtype. A colon (“"”) indicates unreliable values which will not be taken into account for the final spectral types; Col. 15: $\mathrm{H}_{\alpha}$ equivalent widths $(\AA)$. 


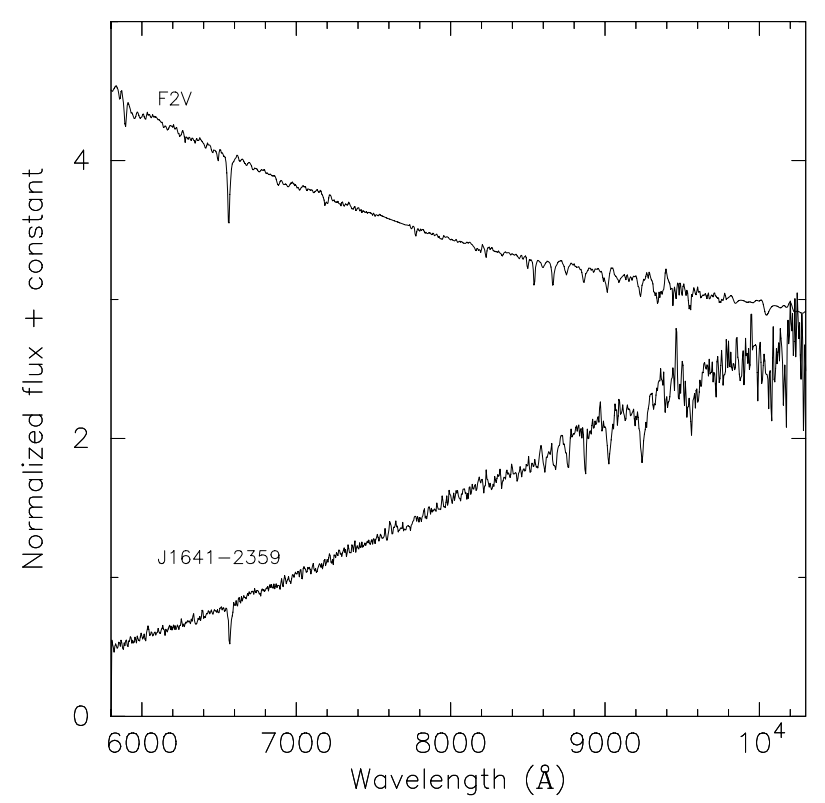

Fig. 5. Comparison between spectra of an F2V dwarf (top) from Pickles (1998) and DENIS-P J 1614-2359 reddened by intervening dust (bottom).

presents comparison between spectra of DENIS-P J1614-2359 and an F2V main sequence star from Pickles (1998). This object is probably in the background of the Rho Ophiuchus molecular complex and reddened by intervening dust as discussed in Crifo et al. (2005).

\section{Summary}

We have presented updated spectroscopic follow-up observations for the DENIS nearby star candidates detected in Papers I and II. Forty one M dwarfs are spectroscopically confirmed in this paper, Crifo et al. (2005) also presented 32M dwarfs. All these stars were pinpointed using the MRPM method that is a robust tool for searching for new nearby ultracool and brown dwarfs in both low and high proper motion surveys in the optical-infrared.

Acknowledgements. This research is carried out based on the DENIS photometry kindly provided by the DENIS consortium. P.-B.N. is grateful to Guy Simon for help during the work. We thank Sébastien Lépine for kindly providing his published spectra. We also thank the referee for many useful comments that clarified and much improved our paper. This research has made use of the SIMBAD and VIZIER databases, operated at CDS, Strasbourg, France.

\section{References}

Bessell, M. S. 1982, PASA, 4, 417

Bessell, M. S., \& Brett, J. M. 1988, PASP, 100, 1134

Bessell, M. S. 1991, AJ, 101, 662

Bessell, M. S. 1999, PASP, 111, 1426

Crifo, F., Phan-Bao, N., Delfosse, X., et al. 2005, A\&A, 441, 653

Cruz, K. L., \& Reid, I. N. 2002, AJ, 123, 2828

Cruz, K. L., Reid, I. N., Liebert, J., et al. 2003, AJ, 126, 2421

Epchtein, N. 1997, in the 2nd DENIS Euroconference, The impact of large scale near-infrared surveys, ed. F. Garzon, et al. (Dordrecht: Kluwer), 15

Gizis, J. E. 1997, AJ, 113, 806

Kirkpatrick, J. D., Reid, I. N., Liebert, J., et al. 1999, ApJ, 519, 802

Lépine, S., Rich, R. M., \& Shara, M. M. 2003a, AJ, 125, 1598

Lépine, S., Shara, M. M., \& Rich, R. M. 2003b, ApJ, 585, L69

Luyten, W. J. 1979, Catalogue of stars with proper motions exceeding 0.'5 annually (LHS), Minneapolis, University of Minnesota

Luyten, W. J. 1980, New Luyten catalog of stars with proper motions larger than Two Tenths of an arcsecond (NLTT), Minneapolis, University of Minnesota

Martín, E. L., Delfosse, X., Basri, G., et al. 1999, AJ, 118, 2466

Phan-Bao, N., Guibert, J., Crifo, F., et al. 2001, A\&A, 380, 590

Phan-Bao, N., Crifo, F., Delfosse, X., et al. 2003, A\&A, 401, 959

Phan-Bao, N., Martín, E. L., Reylé, C., et al. 2005, A\&A, 439, L19

Pickles, A. J. 1998, PASP, 110, 863

Reid, I. N., Hawley, S. L., \& Gizis, J. E. 1995, AJ, 110, 1838

Reid, I. N., Cruz, K. L., Allen, P., et al. 2003, AJ, 126, 3007 